\title{
Rectal perforation and perirectal abscess following stapled hemorrhoidectomy for prolapsed hemorrhoids successfully managed with Endo-SPONGE endoluminal vacuum- assisted wound closure system
}

\author{
Emanuele Rosati, Manuel Valeri, Luigina Graziosi, Lavinia Amato, Stefano Avenia, Annibale Donini \\ Department of Surgery, School of Medicine, University of Perugia, Perugia, Italy
}

Active drains, which work by negative pressure, are commonly used to drain closed airtight wounds. Higher negative pressure is used in vacuum-assisted wound closure dressings. Gastrointestinal leaks may be difficult to treat by surgical approach because of their association with high morbidity and mortality. Recently, endoscopic approaches have been applied with several degrees of success. Most recently, endoluminal vacuum-assisted wound closure (EVAC) has been employed with high success rates in decreasing both morbidity and mortality. In the present paper, the authors describe the successful use of Endo-SPONGE (B. Braun Medical B.V.) EVAC system therapy to drain an open rectal wound, following a perforation occurred during stapled hemorrhoidectomy.

Keywords: Hemorrhoidectomy; Surgical stapling; Rectal fistula; Negative-pressure wound therapy; Case report

\section{INTRODUCTION}

Gastrointestinal leaks can be a burden for a surgeon, especially for esophageal and rectal tract because of their high morbidity and mortality rates. In the past, these cases have been primarily managed by surgical approach; nevertheless, the evolution of alternative therapies such as endoscopic self-expanding metal or plastic stents placement, fibrin glue, or metal clips provide a less invasive method for control and closure of postoperative anastomotic leaks. Unfortunately, these less invasive therapies do not have success. As a result, other minimally invasive options have been developed in order to control leaks and perforations [1,2]. Negative pressure wound therapy (NPWT) is a well-established method

Received: Nov 9, 2020 • Revised: Feb 6, 2021 - Accepted: Feb 7, 2021 Correspondence to: Manuel Valeri, M.D.

Department of Surgery, School of Medicine, University of Perugia, Piazzale Giorgio Menghini, 1, 06129 Perugia PG, Italy

Tel: +39-3401552355

E-mail: valeri.manuel02@gmail.com

ORCID: https://orcid.org/0000-0001-8016-4088

(C) 2022 The Korean Society of Coloproctology

This is an open-access article distributed under the terms of the Creative Commons Attribution NonCommercial License (https://creativecommons.org/licenses/by-nc/4.0) which permits unrestricted noncommercial use, distribution, and reproduction in any medium, provided the original work is properly cited. for treatment of complicated and chronic external wounds. The negative pressure is applied to the wound through a special vacuum-sealed sponge. This sponge continuously or intermittently removes wound secretions or edema, improves microcirculation, and accelerates the growth of granulation tissue. Since its introduction in the late 1990s, the consensus for the routine use of vacuum-assisted wound closure (VAC) system has constantly increased through the years, and it has been adapted for internal use. It has been proven that the use of endoluminal VAC (EVAC) could represent an important alternative therapy in patients with upper and lower intestinal leak who did not respond to standard endoscopic and/or surgical treatment procedures. The method has been adapted from NPWT for complicated ulcers and postoperative wounds. Recent studies have reported the feasibility of an intracavitary and EVAC system to close rectal anastomotic fistulas [2-4]. Any leak after rectal anastomosis can result in pelvic sepsis and sometimes may lead to multiorgan failure. Our single experience has shown that Endo-SPONGE (B. Braun Medical B.V., Melsungen, Germany) system is a safe procedure, well-tolerated by the patient. Moreover, it seems to be a simple and cost-effective therapeutic approach which may significantly enhance recovery thanks to its ability in reducing sepsis's sources in patients undergoing coloproctology surgery. 


\section{CASE REPORT}

A 34-year-old male, with a known past medical history of merely renal lithiasis, underwent stapled hemorrhoidectomy for hemorrhoidal prolapse in another surgical department, complicated by severe intraoperative bleeding and posterolateral rectal perforation accidentally occurred while stapling. He was admitted to our hospital 3 days after surgery. At the admission, the patient was hemodynamically stable, the body temperature was $37.6^{\circ} \mathrm{C}$, and laboratory tests showed mild neutrophilia and slightly increased Creactive protein. CT scan revealed a fluid collection in the presacral and mesorectal spaces, together with an anterolateral right hematoma and retroperitoneal and pelvic air-leak (Fig. 1). No drainage of the collection was performed. Stapled hemorrhoidectomy left a residual open wound measuring $3.0 \times 1.5 \mathrm{~cm}$ in the posterolateral rectal wall. Due to the severe increase in body temperature, on the day after admission, we performed a laparoscopic loop colostomy in order to keep his rectum devoid of any fecal material. Moreover, in order to keep the posterolateral rectal wound empty and to improve the biological tissue regeneration of the rectal wall, we decided to use Endo-SPONGE EVAC system.

\section{Technique device and placement}

EVAC delivers negative pressure to a leak site in the gastrointestinal tract through a nasogastric tube terminating with a polyurethane sponge, named endosponge. The currently available vacuum sponge is the so-called Endo-SPONGE. This is an openpored polyurethane sponge, which is installed transanally after the examination and the rinsing of the abscess cavity with saline solution through a flexible endoscope of outer diameter up to 12 $\mathrm{mm}$. The length and size of the abscess cavity have to be estimated, and the size of the sponge has to be chosen accordingly. When the cavity is too large for 1 sponge, multiple sponges can be

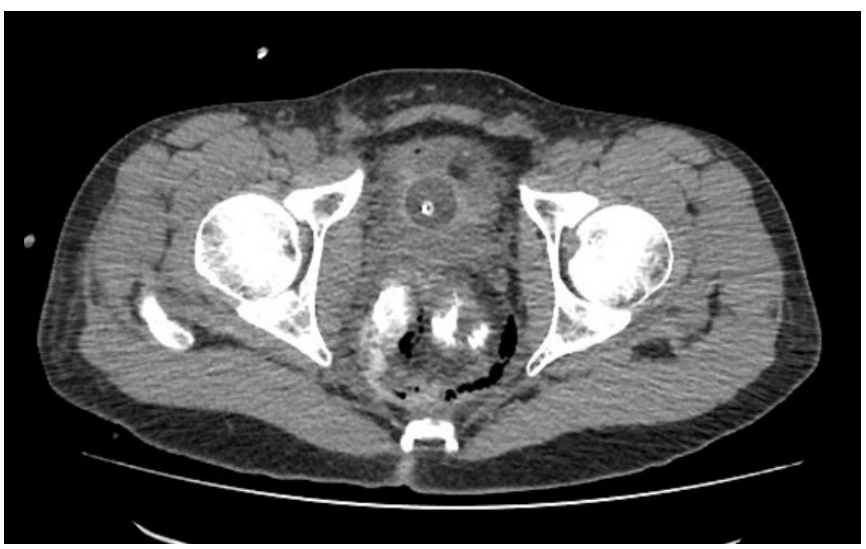

Fig. 1. Postoperative computed tomography scan with gastrografin preparation revealed a fluid mesorectal collection together with a perirectal anterolateral right hematoma, associated with retroperitoneal and pelvic air-leak in proximity to the surgical suture line. placed. After the introduction of the endoscope into the deepest point of the cavity, a plastic tube, positioned over the scope, is advanced into the deepest point of the cavity. After withdrawal of the endoscope, Endo-SPONGE is inserted through the lubricated tube by using a pushing probe while retracting the plastic tube. Next, the sponge is connected to a low vacuum suction bottle, generating a constant negative pressure in the sponge. The correct positioning of the sponge is checked with the endoscope. EndoSPONGE has the ability to collapse the cavity and create a seal. Once it is correctly placed, it provides continuous drainage, promotes granulation, debridement, and rapid cleaning of the wound cavity, mechanically reducing the size of the wound cavity and improving infection control.

In our experience, Endo-SPONGE was placed 10 days after colostomy surgery. The negative pressure itself kept the drain secure and fixed and was well-tolerated by the patient. The drainage system was renewed every 72 hours for 2 times, in order to prevent the growth of the tissue into the sponge and cause painful sponge exchanges [5]. Three days after the last replacement, the VAC system was removed and the patient was discharged in good clinical conditions, with body temperature within normal limit and with decreased inflammatory markers. Postoperative course was unremarkable. After using Endo-SPONGE, we have noticed a narrowing of the cavity and the last bacteriological specimens turned out to be negative. One month after discharge, new CT scans showed complete resolution of the mesorectal fluid collection, together with the disappearance of any air-leak (Fig. 2). Three months later, the patient underwent colon recanalization surgery. Both early outpatient follow-up and 8-month outcome demonstrated excellent recovery.

Written informed consent was obtained for publication of this case report and accompanying images.

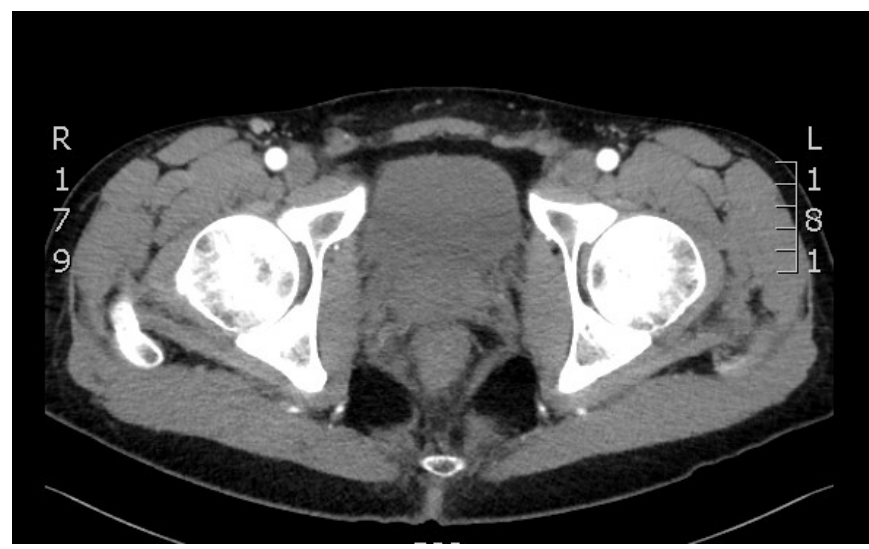

Fig. 2. Computed tomography scan 1 month after discharge showed complete resolution of the mesorectal fluid collection, significant reduction of mesorectal fascia edema, and disappearance of intraabdominal air-leak. R, right; L, left. 


\section{DISCUSSION}

Stapled hemorrhoidectomy is a relatively new technique that was developed in Italy in 1997. It is considered to be more physiological because it lifts hemorrhoidal tissue back to its original place [6]. Complications which may occur in $15 \%$ of patients undergoing stapled hemorrhoidectomy include bleeding, pain, anorectal stenosis, recurrence, urgency, retention of urine, incontinence, fissure, anorectal sepsis, and rectovaginal fistulas. Even though uncommon, pelvic sepsis following surgery for prolapsed hemorrhoids can be life-threatening. The exact mechanism of pelvic sepsis is not clearly known. It may involve incorporation of bacteria during stapling into the rectal wall. Therefore, prophylactic antibiotics may decrease the incidence of pelvic sepsis [6-8].

VAC therapy is a high negative pressure therapy, requiring a pressure of $-125 \mathrm{mmHg}$ to be effective. Getting a seal around mucosa, which theoretically can cause pressure necrosis, can be difficult and it is not licensed for rectal wounds. Endo-SPONGE EVAC system is commercially available for the treatment of anastomotic leaks [6].

The first report about EVAC was published in 2008 by Wedemeyer et al. [9]. They described 2 patients with anastomotic leak after intrathoracic esophagogastrostomy and esophagojejunostomy due to esophagus carcinoma.

Durai and Ng [6] indicated that the success of the novel combination of the sponge of the VAC (KCI Medical Ltd., Kidlington, UK) dressing with sealed Redivac system (Unomedical, Birkerod, Denmark), in the drainage of an open rectal wound, depended on the safety of a proven, sealed system associated with the innovation of a tailored, homemade seal.

A later paper by Durai and $\mathrm{Ng}$ [10] demonstrated the feasibility of their novel procedure in a patient who suffered from a perirectal abscess following stapled hemorrhoidopexy; in our case, due to a persistent vacuum loss, the Redivac drainage system was switched to a J-VAC (Ethicon Inc., Somerville, NJ, USA)/VAC sponge combination, where the vacuum can be easily recreated without requiring to change the reservoir. When the presence of a severe inflammation involving tissues is noticed, it is not advisable to stitch the mucosal defect in the rectum, and the only way to keep the rectal wound dry is a constant, low negative pressure therapy [10].

Anastomotic leak, following colorectal surgery, is a severe and feared complication, difficult to manage with a surgical approach. Recently, endoscopic treatments have been performed with several degrees of success. In case of anastomotic leak, large cavities might become persistent [5]. Therefore, immediate treatment is mandatory [11]. Vacuum-based system promotes the closure of the cavity through the application of negative pressure into the sponge, which ensures continuous drainage and keeps the infection from spreading; it allows narrow wound drainage, closure of several defects, and tissue granulation itself. Due to the presence of chronic sepsis and fibrotic tissue, all invasive procedures are technically difficult and complicated [5]. As a matter of fact, EVAC was found to be an effective and promising treatment for wound drainage and anastomotic leaks and sometimes happens to be better than surgical approach or stent placement [12]. Furthermore, EVAC has been proven to have high success rates in decreasing both morbidity and mortality in the treatment of perforations and leaks of the gastrointestinal tract. It can also be considered a cost-effective procedure, well-accepted by patients, managed in outpatient clinics as well [2]. It has been reported that this therapy is effective in patients following neoadjuvant chemoradiotherapy and with additional chemoradiotherapy [13]. In the present paper, we reported the case of a 34-year-old male patient, undergone stapled hemorrhoidectomy, presenting with posterolateral rectal perforation and related presacral and mesorectal fluid collection. In our experience, EVAC therapy, in an uncommon case of treatment following stapled hemorrhoidopexy, combined with laparoscopic colostomy, significantly enhanced recovery by helping to drain the abscess, close the defect and promote tissue granulation with a proper control of the infection.

In some departments and clinics transanal EVAC therapy, endoscopic transanal vacuum-assisted rectal drainage or endosponge therapy has been established as the routine treatment for patients with major rectal anastomotic leak without sepsis. The EVAC treatment was found to be effective in patients with leak following neoadjuvant chemoradiotherapy, iatrogenic perforations, and anastomotic fistulas, too $[2,4,13]$.

Endo-SPONGE (B. Braun Medical B.V.) treatment for large colorectal anastomotic leak following anterior rectal resection, performed in suitable patients, represents a successful and safe approach with long-term efficacy. The reduction in wound closure time, mild-to-moderate discomfort, and possibly shorter hospitalization suggest that Endo-SPONGE treatment can be a prominent therapeutic regimen with adequate patient acceptance. On the other hand, major limitations of the EVAC system are represented by the necessity of repeated endoscopic procedures and constant presence of well-trained staff [14]. Some authors suggested that the EVAC method may be ineffective in the course of sepsis [15]. As we have been able to appreciate in our case, timing is very important and early treatment has a much higher success rate [11]. Further, large cohort studies need to be performed to establish the applicability and effectiveness of EVAC before routine widespread use can be recommended.

According to our single case experience, the EVAC treatment of rectal perforation and perirectal abscess following stapled hemorrhoidectomy for prolapsed hemorrhoids could be one of the most promising applications of vacuum-assisted closure in coloproctology.

\section{CONFLICT OF INTEREST}

No potential conflict of interest relevant to this article was reported. 


\section{FUNDING}

None.

\section{ORCID}

Emanuele Rosati, https://orcid.org/0000-0003-2768-5989

Manuel Valeri, https://orcid.org/0000-0001-8016-4088

\section{REFERENCES}

1. Leeds SG, Mencio M, Ontiveros E, Ward MA. Endoluminal vacuum therapy: how I do it. J Gastrointest Surg 2019;23:1037-43.

2. Borejsza-Wysocki M, Szmyt K, Bobkiewicz A, Malinger S, Świrkowicz J, Hermann J, et al. Endoscopic vacuum-assisted closure system (E-VAC): case report and review of the literature. Wideochir Inne Tech Maloinwazyjne 2015;10:299-310.

3. Krokowicz L, Borejsza-Wysocki M, Mackiewicz J, Drews M. 10 years of negative pressure wound therapy (NPWT): evolution of indications for its use. Negat Press Wound Ther J 2014;1:27-32.

4. Weidenhagen R, Gruetzner KU, Wiecken T, Spelsberg F, Jauch KW. Endoscopic vacuum-assisted closure of anastomotic leakage following anterior resection of the rectum: a new method. Surg Endosc 2008;22:1818-25.

5. Bemelman WA. Vacuum assisted closure in coloproctology. Tech Coloproctol 2009;13:261-3.

6. Durai R, Ng PC. Perirectal abscess following procedure for prolapsed haemorrhoids successfully managed with a combination of VAC sponge and Redivac systems. Tech Coloproctol 2009;13: 307-9.

7. Shao WJ, Li GC, Zhang ZH, Yang BL, Sun GD, Chen YQ. Systematic review and meta-analysis of randomized controlled trials comparing stapled haemorrhoidopexy with conventional haem- orrhoidectomy. Br J Surg 2008;95:147-60.

8. McCloud JM, Jameson JS, Scott AN. Life-threatening sepsis following treatment for haemorrhoids: a systematic review. Colorectal Dis 2006;8:748-55.

9. Wedemeyer J, Schneider A, Manns MP, Jackobs S. Endoscopic vacuum-assisted closure of upper intestinal anastomotic leaks. Gastrointest Endosc 2008;67:708-11.

10. Durai R, Ng PC. Novel combination combining J-Vac and VAC sponge for draining a rectal wound. Acta Chir Belg 2010;110:4024.

11. van Koperen PJ, van Berge Henegouwen MI, Rosman C, Bakker CM, Heres P, Slors JF, et al. The Dutch multicenter experience of the endo-sponge treatment for anastomotic leakage after colorectal surgery. Surg Endosc 2009;23:1379-83.

12. Schniewind B, Schafmayer C, Voehrs G, Egberts J, von Schoenfels W, Rose T, et al. Endoscopic endoluminal vacuum therapy is superior to other regimens in managing anastomotic leakage after esophagectomy: a comparative retrospective study. Surg Endosc 2013;27:3883-90.

13. von Bernstorff W, Glitsch A, Schreiber A, Partecke LI, Heidecke CD. ETVARD (endoscopic transanal vacuum-assisted rectal drainage) leads to complete but delayed closure of extraperitoneal rectal anastomotic leakage cavities following neoadjuvant radiochemotherapy. Int J Colorectal Dis 2009;24:819-25.

14. Mussetto A, Arena R, Buzzi A, Fuccio L, Dari S, Brancaccio ML, et al. Long-term efficacy of vacuum-assisted therapy (Endo$\mathrm{SPONGE}^{\circledR}$ ) in large anastomotic leakages following anterior rectal resection. Ann Gastroenterol 2017;30:649-53.

15. Bludau M, Hölscher AH, Herbold T, Leers JM, Gutschow C, Fuchs $\mathrm{H}$, et al. Management of upper intestinal leaks using an endoscopic vacuum-assisted closure system (E-VAC). Surg Endosc 2014;28:896-901. 